\title{
Evaporation of Sessile Water Droplets in Presence of Contact Angle Hysteresis
}

\author{
S. Semenov ${ }^{1}$, V.M. Starov ${ }^{1 *}$, R.G. Rubio ${ }^{2}$, H. Agogo ${ }^{2}$, M.G. Velarde ${ }^{3}$ \\ ${ }^{1}$ Dept. of Chemical Engineering, Loughborough University, LE11 3TU Loughborough, UK \\ ${ }^{2}$ Dept. of Quimica Fisica I, Universidad Complutense, 28040 Madrid, Spain \\ ${ }^{3}$ Instituto Pluridisciplinar, Universidad Complutense, 28040 Madrid, Spain
}

\begin{abstract}
In this paper we present a theory describing the diffusion limited evaporation of sessile water droplets in presence of contact angle hysteresis. Theory describes two stages of evaporation process: (I) evaporation with a constant radius of the droplet base; and (II) evaporation with constant contact angle. During stage (I) the contact angle decreases from static advancing contact angle to static receding contact angle, during stage (II) the contact angle remains equal to the static receding contact angle. Universal dependences are deduced for both evaporation stages. Obtained universal curves are validated against available in the literature experimental data.
\end{abstract}

Keywords and phrases: evaporation, sessile droplets, contact angle hysteresis

Mathematics Subject Classification: 76D05, 76D45, 76R50

\section{Introduction}

Evaporation of a sessile liquid droplet is important in many industrial applications. Drying droplets are used in molecular combing process $[3,13,32]$ (e.g. aligning and stretching of DNA molecules); in cooling and heat exchanging systems [31]. Processes like evaporation and condensation are the basic mechanism in micro-heat pipes [28] and spray cooling of electronic components [8]. Evaporation also governs the deposition of suspended colloidal particles in an evaporating droplet, which is important for coating [30], painting and printing [33,34,39]. 2D and 3D inkjet printing [16, 18, 29,33,39] of functional materials (e.g. pixel arrays [18,29], metal micro structures [33], printed electronics, printed MEMS devices [16]) using colloidal suspensions is of great interest nowadays. Tissue engineering with the aid of inkjet printers (bioprinting) [6] is promising area of researches. Ink drying regimes play an important role for a high-quality printing [34].

From the scientific point of view the problem of evaporation of a sessile droplet is of a substantial interest because of singularity problems arising at the three-phase contact line: (i) a singularity of evaporation flux due to an incompatibility of boundary conditions at the liquid-gas interface and at the solid-liquid and solid-gas interfaces; (ii) the viscous stress singularity due to no-slip boundary condition at the solid surface which is usually used in continuum hydrodynamics. The solution of those problems on

${ }^{*}$ Corresponding author. E-mail: V.M.Starov@lboro.ac.uk 
a microscale level and obtaining of corresponding macroscopic boundary conditions is one of the research goals in this field.

It has been established $[5,41]$ that in the presence of contact angle hysteresis the evaporation of a sessile droplet in non-saturated vapour atmosphere can go through four consequent stages: (0) a spreading until the value of static advancing contact angle, $\theta_{a d}$, is reached; (I) evaporation proceeds with a constant contact area and decreasing contact angle, $\theta$, until the contact angle reaches the receding value, $\theta_{r}$; (II) evaporation with a constant contact angle, $\theta_{r}$, and decreasing radius of the contact line, $L$; (III) evaporation with decrease of both $L$ and $\theta$ until droplet disappears. During the first stage (0) the evaporation can be neglected and, hence, can be described using conventional hydrodynamic approach [43]. The mechanism of evaporation at stage (III) is yet to be understood. Those two stages are not considered below.

The evaporation of sessile droplets has been studied for many years. Deegan et al. [11,12] showed that the density of vapour flux, $j$, is not uniformly distributed over the surface of a sessile droplet. The latter non-uniformity generates flow inside the droplet, which transports suspended solid particles to the edge of the droplet thus leading to the droplet pinning and ring-like stain formation. Cazabat et al. [24] showed that the vapour flux density, $j$, over the droplet is inversely proportional to the radius of the contact line, $L$.

Different studies [19-23,35] give similar equation for the evaporation rate of a sessile droplet:

$$
\frac{d V}{d t}=-2 \pi \frac{D M}{\rho}\left(c_{\text {sat }}\left(T_{\text {surf }}\right)-H c_{\text {sat }}\left(T_{\infty}\right)\right) F(\theta) L,
$$

where $V$ is the droplet volume, $t$ is time, $D, \rho$, and $M$ are vapour diffusivity in the air, density of the liquid and the molar mass, respectively; $H$ is humidity of ambient air; $T_{\text {surf }}$ is the temperature of the droplet-air interface and $T_{\infty}$ is the temperature of the ambient air; $c_{\text {sat }}\left(T_{\text {surf }}\right)$ and $c_{\text {sat }}\left(T_{\infty}\right)$ are the molar concentrations of saturated vapour at the corresponding temperature; $F(\theta)$ is a function of the contact angle, $\theta$, with value 1 at $\theta=\pi / 2$. Eq. (1.1) ignores the temperature distribution along the droplet-air interface.

Evaporation of droplets with contact angles $\theta<120^{\circ}$ was studied by Girard et al. [19-23] and Hu and Larson [25-27]. The former investigated the influence of substrate heating [22,23], air humidity [22] and Marangoni convection [19]. They concluded that the effect of Marangoni convection is negligible in comparison with the influence of other physical phenomena. Hu and Larson investigated the process of particle deposition and ring-like stain formation during droplet evaporation [25-27].

Dunn et al. [14] proposed a mathematical model for the quasi-steady diffusion limited evaporation of a thin axisymmetric sessile droplet of liquid with a pinned contact line. They studied the dependence of saturated vapour pressure on the temperature of the cooled droplet's surface, although the Marangoni convection was not considered. The predictions of their model are in good qualitative, and in some cases also quantitative, agreement with their experimental results. Later Dunn et al. [15] demonstrated a strong influence of thermal conductivity of the substrate on the evaporation of a pinned sessile droplet. They included into their model the buoyancy of water vapour in the atmosphere, and used Newton's law of cooling on the unwetted surface of the substrate, which introduced an empirical coefficient to the system of equations. By choosing the value of that empirical coefficient they managed to reach the quantitative agreement between their computer simulations and experimental data.

Ristenpart et al. [37] investigated the influence of the substrate conductivity on a reversal of Marangoni convection within evaporating sessile droplets. They neglected the heat conductivity of the air and used predefined distribution of evaporation flux over the droplet surface. Despite all the assumptions made by the authors, their quantitative criterion for the circulation direction has been confirmed experimentally [37].

Bhardwaj et al. [4] solved numerically a very complex problem of drying colloidal droplets and deposits formation. The interaction of the liquid-air interface with the peripheral deposit of colloidal particles, depinning and receding of the contact line were simulated by the authors. The formation of different deposit patterns obtained experimentally was explained well by their simulations [4]. 
Craster et al. [9] studied evaporation of sessile droplets to ambient vapour. They used kinetic model of evaporation. Their model accounted for Derjaguin's (disjoining) pressure including structural forces due to presence of nanoparticles in the liquid. The main purpose of their study was the understanding of the influence of nanoparticles concentration on the processes of droplets spreading and evaporation. Their results indicated that evaporative flux is not diverging at the three phase contact line, but still has a maximum over there. Craster et al. [9] demonstrated also a variety of different behaviours of the system in question: spreading, retraction, contact line pinning, and "terrace" formation.

Ajaev [2] has developed a mathematical model for the spreading of a thin volatile liquid droplet on a uniformly heated surface. He used lubrication-type approximation and one-sided model, when density, dynamic viscosity, and heat conductivity of gas phase are very small compared to those of liquid. He avoided singularity problems at the three-phase contact line by introducing the action of generalized disjoining pressure which depends on both thickness of the liquid layer and slope of the liquid-gas interface. Results of that work show high but finite evaporation flux in the area of three-phase contact line, as well as rapid change of the interface curvature in that area. The author concluded that the droplet dynamics is governed mostly by competition between evaporation and capillary spreading.

Rednikov et al. [36] studied the microstructure of a contact line formed by a liquid and its pure vapour for a perfectly wetted superheated smooth substrate with a disjoining pressure in the form of a positive inverse cubic law. They took a spreading coefficient as an independent parameter in their study. Results show that regime of a truncated liquid microfilm on a solid surface can be thermodynamically more stable than the regime of an extended microfilm (covering the whole solid surface), even if the spreading coefficient is still positive (perfect wetting).

Sultan et al. [44] constructed a system of equations, which describes the evaporation of a thin liquid layer. They applied the lubrication approximation and combined the diffusion limited evaporation with the thermodynamic rate of transfer across the liquid-gas interface. Linear stability analysis of the obtained system of equations helped authors to find a correlation between the instability of a uniform liquid layer and the festoon instability of an evaporating droplet.

Previously [42] we simulated the evaporating droplet of a pure liquid taking into account: the heat transfer through the whole system, vapour diffusion into the ambient air, and Marangoni convection inside of the droplet. Computer simulations allowed us to investigate the influence of latent heat of vaporization and thermal Marangoni convection on the evaporation rate, $J$. It was shown that one of the important parameters influencing the process of evaporation is the average temperature, $T_{a v}$, of the surface of an evaporating droplet.

Non-physical singularities, discussed above, appear only within the mesh cells adjacent to the three-phase contact line. The influence of those singularities is reduced by refining the computational mesh around the contact line, thus minimizing the size of cells with singularities. Future work implies using some method for alleviation of the singularities [38], for instance, by introducing a precursor film.

Below we deduce a universal law of evaporation for sessile droplets of pure water in presence of contact angle hysteresis. This law of evaporation is based on the results of computer simulations presented in [42].

\section{Theory}

\subsection{Problem statement}

Droplets in question are supposed to have size $L \ll a$, where $a=\sqrt{\gamma / \rho g}$ is the capillary length, $\gamma$ is the surface tension of liquid-air interface, $\rho$ is the liquid density, and $g$ is the gravitational acceleration. For water droplets $a \approx 2.7 \mathrm{~mm}$. Thus gravity force can be neglected and then liquid-air interface will have the shape of a spherical cap (Figure 1) as a direct consequence of the action of surface tension. The evaporation process is governed by diffusion in the ambient air, what imposes the lower limit for the droplet size which is of order of $10^{-7} \mathrm{~m}$ [42]. According to the Kelvin equation [17] the concentration of saturated vapour above the droplet's surface depends on the curvature of this surface. Estimations show 


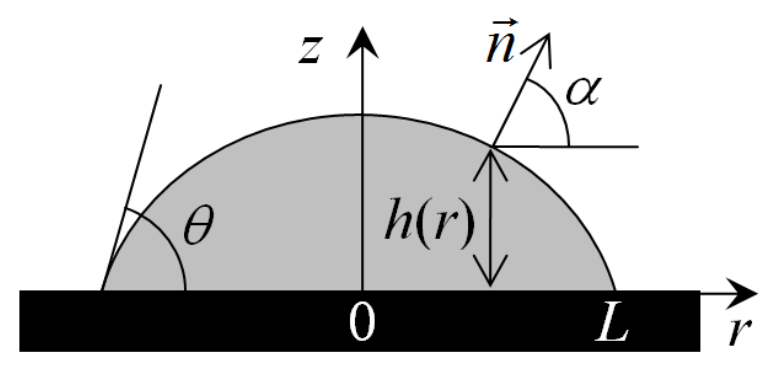

Figure 1. Droplet parameters in the case $\theta<\frac{\pi}{2}$ : contact line radius $L$, height $h(r)$, contact angle $\theta$ and normal unit vector $\vec{n}$.

that for droplet size more than $10^{-7} \mathrm{~m}$ variation in the vapour concentration at the liquid-gas interface due to surface curvature is less than $1 \%$. Therefore, this variation of the concentration of the saturated vapour is not taken into account below. The problem is solved using a steady-state approximation, taking into account Marangoni convection, latent heat of vaporization and heat transfer across the whole system in question.

\subsection{Preliminary computer simulations}

We performed computer simulations [42] of heat and mass transfer in the system of an evaporating sessile droplet on the surface of a smooth solid substrate, and obtained the dependence of total evaporation flux, $J$, on system parameters. Comparison of our results [42] with the isothermal dependence $J(\theta)$, obtained by Picknet and Bexon [35], showed that total evaporation flux, $J$, obtained in our non-isothermal computer simulations is well described by the formula (2.1), which is different from that for the isothermal case only by substituting the average temperature of the droplet surface, $T_{a v}$, instead of the ambient one, $T_{\infty}$, into the formula for a saturated vapour concentration at the liquid-gas interface.

$$
J=F(\theta) \cdot J_{\pi / 2}\left(L, T_{a v}\right)
$$

where:

$T_{a v}=\frac{1}{S} \int_{S} T d S$ is the average temperature of the droplet surface, $S$;

$F(\theta)= \begin{cases}\left(0.6366 \cdot \theta+0.09591 \cdot \theta^{2}-0.06144 \cdot \theta^{3}\right) / \sin \theta, & \theta<\pi / 18 \\ \left(8.957 \mathrm{e}-5+0.6333 \cdot \theta+0.116 \cdot \theta^{2}-0.08878 \cdot \theta^{3}+0.01033 \cdot \theta^{4}\right) / \sin \theta, & \theta \geq \pi / 18\end{cases}$

where contact angle, $\theta$, is in radians. $F(\theta)$ is the function derived by R.G. Picknett and R. Bexon [35] for the isothermal evaporation and later used by F. Schonfeld et al. [40];

$$
J_{\pi / 2}\left(L, T_{\text {surf }}\right)=2 \pi D\left(c_{\text {sat }}\left(T_{\text {surf }}\right)-c_{\infty}\right) L .
$$

$J_{\pi / 2}\left(L, T_{\text {surf }}\right)$ is the analytical solution of the following problem (see Figure 2): evaporating sessile droplet with contact angle $\theta=\pi / 2$; molar concentration, $c_{\text {sat }}\left(T_{\text {surf }}\right)$, of saturated vapour at the surface of the droplet; uniform temperature of the droplet surface, $T_{\text {surf }}$; molar concentration, $c_{\infty}=H c_{\text {sat }}\left(T_{\infty}\right)$, of vapour in the ambient air far away from the droplet; and diffusion coefficient, $D$, of vapour in the air.

Taking into account the result of computer simulations [42] expressed according to Eq. (2.1), we can rewrite Eq. (1.1) as:

$$
\frac{d V}{d t}=-\beta F(\theta) L
$$

where

$$
\beta=2 \pi \frac{D M}{\rho}\left(c_{s a t}\left(T_{a v}\right)-c_{\infty}\right) .
$$




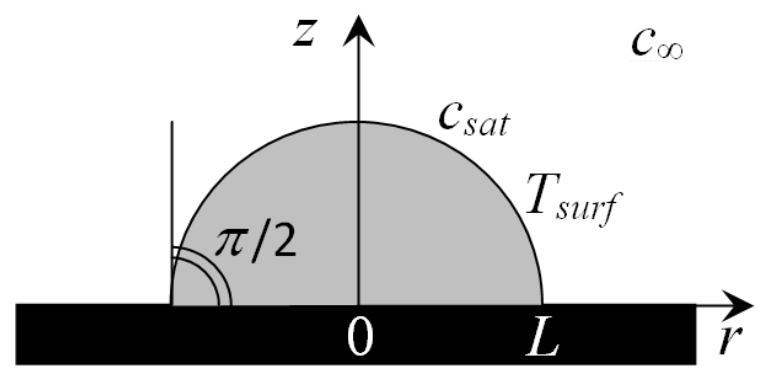

FiguRE 2. Illustration of a problem with contact angle $\theta=\pi / 2$, which has an analytical solution for total evaporation flux: $J_{\pi / 2}\left(L, T_{\text {surf }}\right)$

The latter equation shows that for a pure fluid parameter $\beta$ depends on the average temperature, $T_{a v}$, of the droplet surface.

\subsection{Average temperature of the surface of a sessile droplet}

Let us estimate the variation of the average temperature of the droplet surface, $T_{a v}$, in the course of droplet evaporation. Our computer simulations show (see Figure 3) the dependence of temperature of an aqueous drop $T_{a v}-T_{\infty}$ on the substrate temperature, $T_{s}$, far away from the droplet; on the thermal conductivity, $k_{s}$, of the substrate and on the contact angle. Figure 3 clearly shows that dependence of $T_{a v}$ on contact angle, $\theta$, is much less pronounced than its dependence on $T_{s}$ and $k_{s}$.

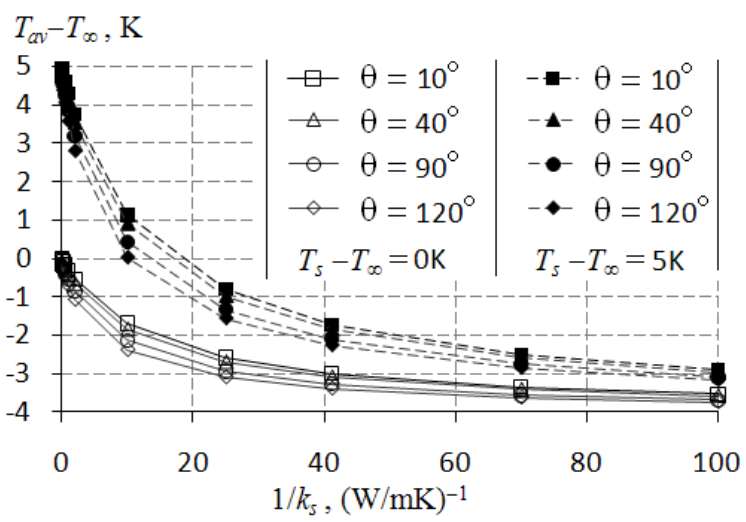

FiguRE 3. Simulated dependences of average temperature, $T_{a v}$, of the droplet's surface on the reciprocal of thermal conductivity of the substrate, $k_{s}$, and droplet contact angle, $\theta$. Liquid is water. Both latent heat of vaporization and Marangoni convection are taken into account. $T_{s}$ and $T_{\infty}$ are the temperatures of the substrate and of the air, respectively far away from the droplet. Humidity $H=70 \% . L=1 \mathrm{~mm}, T_{\infty}=293 \mathrm{~K}$.

We conclude from Figure 3 that if heat conductivity and temperature of the substrate remain constant during the evaporation of a pinned droplet, then the average temperature, $T_{a v}$, of the droplet surface is almost constant. It can only vary within the range of 1 degree (Figure 3 ) in the course of evaporation due to weak dependence on the contact angle.

Experiments by S. David et al. [10] have shown that temperature in the bulk of a sessile evaporating droplet substantially depends on the thermal properties of the substrate and the rate of evaporation. 
Their measurements (Figure 4) clearly show that temperature of an evaporating droplet is different from the ambient temperature and almost constant in the course of evaporation. We assume that the constancy over time of the droplet bulk temperature (Figure 4) also implies the constancy over time of its average surface temperature.

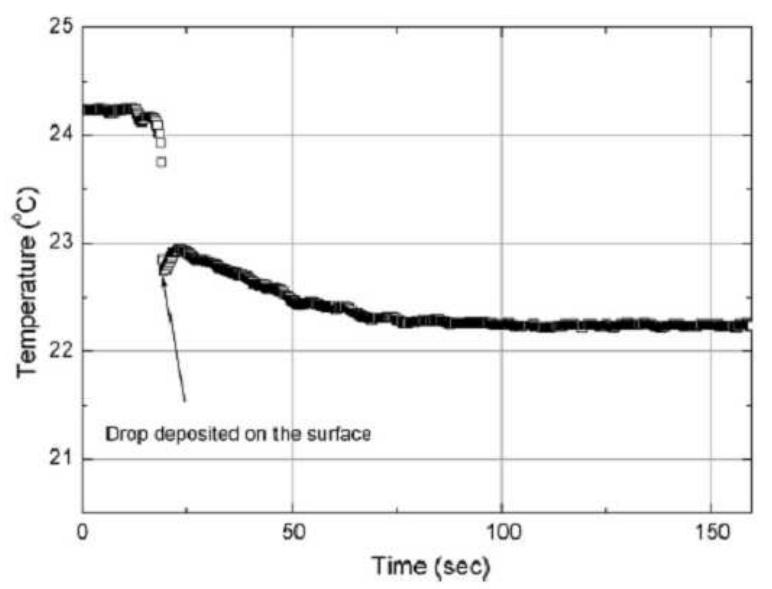

Figure 4. Evolution of temperature inside the droplet after water droplet is deposited on PTFE substrate. Redrawn from [10].

Above mentioned arguments support the assumption of relative constancy of the average temperature of the droplet surface in the course of evaporation:

$$
T_{a v} \approx \text { const. }
$$

Figure 3 shows that the influence of the substrate's outer temperature, $T_{s}$, on the average temperature of the droplet's surface, $T_{a v}$, vanishes as thermal conductivity of the substrate, $k_{s}$, tends to zero. That is, there exists a limit for the temperature drop, $T_{a v}-T_{\infty}$, which is identical for all curves in the Figure 3 . This limit must depend on the temperature, $T_{\infty}$, and humidity, $H$, of the ambient air. Let us calculate this limit.

When thermal conductivity of the substrate, $k_{s}$, is zero, then liquid droplet and air are thermally insulated from the substrate. In this particular case the vaporization process is maintained by the heat flux from the ambient air. Let us show that in case of contact angle $\theta=\pi / 2$, and $k_{s}=0$ an analytical solution of the problem under consideration exists. Absence of normal heat and mass fluxes at the flat surface of the substrate allows us considering this surface as a plane of symmetry. The latter shows that our problem has actually a spherical symmetry. The solution of the latter problem depends only on radial coordinate, $r$, and gives uniform evaporation and heat fluxes along the droplet surface. As the heat sink (due to vaporization) is distributed over the droplet's surface, the spherical symmetry implies uniform temperature field inside the droplet and on its surface. As a result: there are no heat fluxes inside the droplet, and no Marangoni convection. Solving Laplace equations for vapour concentration, $c$, and temperature, $T$, in infinite air domain gives the following solutions:

$$
\begin{gathered}
c=H c_{\text {sat }}\left(T_{\infty}\right)+\frac{c_{\text {sat }}\left(T_{\text {surf }}\right)-H c_{\text {sat }}\left(T_{\infty}\right)}{r} L, \\
T=T_{\infty}+\frac{T_{\text {surf }}-T_{\infty}}{r} L
\end{gathered}
$$

where $r$ is the radial coordinate in the spherical system of coordinates. 
Let us analyse the stability of such evaporation regime $\left(k_{s}=0, \theta=\pi / 2\right)$ by considering a small local disturbance of evaporation flux. If evaporation rate is locally increased/decreased then due to increased/decreased heat consumption (latent heat) the latter results in a local temperature decrease/increase. It leads to a decrease/increase of a local concentration of saturated vapour, and as a consequence, to a local decrease/increase of evaporation rate. Thus an initial small disturbance in the system is suppressed. In addition, local Marangoni stresses always appear on the surface where local temperature gradient appears: it generates liquid flows along the surface, which contribute to elimination of temperature non-uniformity. Thus, according to the above mentioned qualitative reasoning, we come to a conclusion that the system in question is in a stable regime of the quasi-steady process of evaporation.

Using the obtained solution, Eqs. (2.5) and (2.6), we can calculate the density of molar vapour flux, $\left.j_{c}\right|_{r=L}$, and density of heat influx, $\left.j_{h}\right|_{r=L}$, on the surface:

$$
\begin{gathered}
\left.j_{c}\right|_{r=L}=-\left.D \frac{\partial c}{\partial r}\right|_{r=L}=D \frac{c_{\text {sat }}\left(T_{\text {surf }}\right)-H c_{\text {sat }}\left(T_{\infty}\right)}{L} \\
\left.j_{h}\right|_{r=L}=\left.k_{a} \frac{\partial T}{\partial r}\right|_{r=L}=-k_{a} \frac{T_{\text {surf }}-T_{\infty}}{L}
\end{gathered}
$$

where $k_{a}$ is the thermal conductivity of the surrounding air. Boundary condition for fluxes gives:

$$
\left.j_{c}\right|_{r=L} \Lambda=\left.j_{h}\right|_{r=L}
$$

where $\Lambda$ is the latent heat of vaporization in $\mathrm{J} / \mathrm{mol}$. It follows from Eqs. $(2.7)-(2.9)$ that

$$
c_{\text {sat }}\left(T_{\text {surf }}\right)=H c_{\text {sat }}\left(T_{\infty}\right)+\frac{k_{a}}{D \Lambda}\left(T_{\infty}-T_{\text {surf }}\right) .
$$

We have taken the tabulated values of saturation pressure of water vapour (2337 $\mathrm{Pa}$ ) and latent heat of vaporization $\left(44320 \mathrm{~J} / \mathrm{mol}\right.$ ) at temperature $20^{\circ} \mathrm{C}$ [1], and have applied the Clausius-Clapeyron equation [42] to express the dependence of molar concentration of saturated vapour, $c_{\text {sat }}$ (in $\mathrm{mol} / \mathrm{m}^{3}$ ), on temperature, $T$. This dependence, $c_{\text {sat }}(T)$, can be approximated for the temperature range $(273 \mathrm{~K}<T<303$ $\mathrm{K})$ with the following quadratic equation:

$$
c_{\text {sat }}(T) \approx 1.1951 \cdot 10^{-3} \cdot T^{2}-6.4255 \cdot 10^{-1} \cdot T+86.636,
$$

where temperature, $T$, is in Kelvin and concentration, $c_{s a t}$, is in $\mathrm{mol} / \mathrm{m}^{3}$. Taking into account approximation (2.11), Eq. (2.10) can be easily solved with respect to the unknown $T_{\text {surf }}$ within range $(273 \mathrm{~K}$ $\left.<T_{\text {surf }}<303 \mathrm{~K}\right)$. We denote the root of the Eq. (2.10) as $\left.T_{\text {surf }}\right|_{\substack{k_{s}=0 \\ \theta=\pi / 2}}$, which is the temperature of the droplet's surface in case of contact angle $\theta=\pi / 2$ and thermal conductivity of the substrate $k_{s}=0$.

Thus, using Eq. (2.10) we have calculated the value of $\left.T_{\text {surf }}\right|_{\substack{k_{s}=0 \\ \theta=\pi / 2}}$ for the humidity $H=70 \%$ and temperature of the ambient air $T_{\infty}=293 \mathrm{~K}$ (as in the Figure 3$)$. The result is $\left.T_{\text {surf }}\right|_{\substack{k_{s}=0 \\ \theta=\pi / 2}}-T_{\infty}=-3.9 \mathrm{~K}$. This temperature drop is the limit attained by the curves in Figure 3 as the $k_{s}^{-1}$ tends to infinity. Using this temperature drop we can write an expression for dimensionless temperature:

$$
\chi=\frac{T_{a v}-\left.T_{\text {surf }}\right|_{\substack{k_{s}=0 \\ \theta=\pi / 2}}}{T_{s}-\left.T_{\text {surf }}\right|_{\substack{k_{s}=0 \\ \theta=\pi / 2}}}
$$

Figure 5 shows the plot of $\chi$ versus dimensionless thermal conductivity $k_{w} /\left(k_{a}+k_{s}\right)$, where subscripts $w$, 


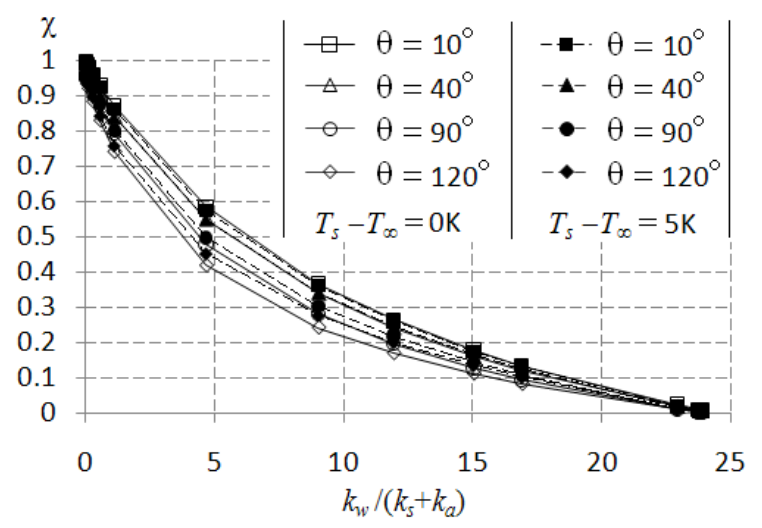

Figure 5. The same as Figure 3 using non-dimensional temperature Eq. (2.12).

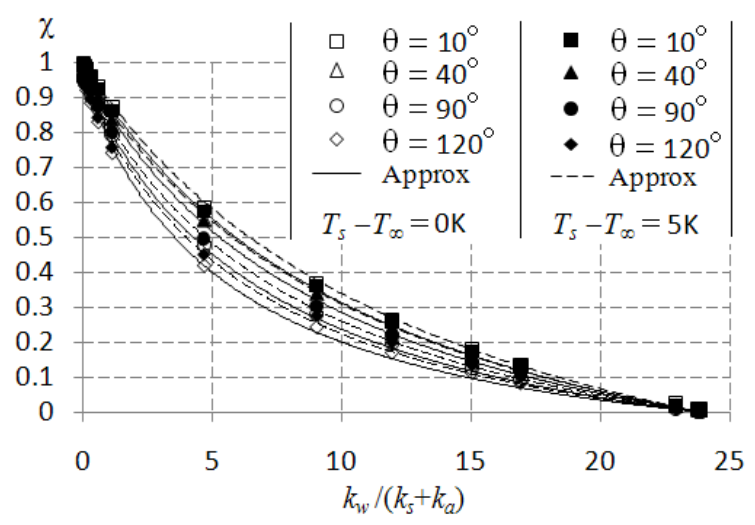

FiguRE 6. The same as Figure 5 using approximating curves Eqs. (2.13) and (2.14).

$a$, and $s$ correspond to "water", "air", and "substrate" respectively. Figure 5 shows that for contact angles $10^{\circ}$ and $40^{\circ}$ there is almost no difference between calculated values of $\chi$ for two specific temperatures of the substrate, $T_{s},\left(T_{s}\right.$ is specified far away from the droplet). But for contact angles $90^{\circ}$ and $120^{\circ}$ there is small difference: in the case $T_{s}-T_{\infty}=5 \mathrm{~K}$ the value of $\chi$ is slightly higher than that in the case $T_{s}-T_{\infty}=0 \mathrm{~K}$.

Simulated results in Figure 5 in the case $T_{s}-T_{\infty}=0 \mathrm{~K}$ can be approximated by the following semi-empirical equation ( $\theta$ is in radians):

$$
\left.\chi\left(k_{s}, \theta\right)\right|_{T_{s}-T_{\infty}=0 \mathrm{~K}} \approx \frac{1}{1+F(\theta)\left[\left(k_{a} / k_{w}\right) \sin \theta+\left(k_{a} / k_{s}\right)(\sin \theta-0.75 \cdot \theta+4.61)\right]},
$$

with an approximation error: $\Delta \chi=\Delta T /\left(T_{s}-\left.T_{\text {surf }}\right|_{\substack{k_{s}=0 \\ \theta=\pi / 2}}\right)<0.02$.

Simulated results in Figure 5 in the case $T_{s}-T_{\infty}=5 \mathrm{~K}$ can be approximated by the next equation $(\theta$ is in radians):

$$
\left.\chi\left(k_{s}, \theta\right)\right|_{T_{s}-T_{\infty}=5 \mathrm{~K}} \approx \frac{1}{1+F(\theta)\left[\left(k_{a} / k_{w}\right) \sin \theta+\left(k_{a} / k_{s}\right)(\sin \theta-0.75 \cdot \theta+4.09)\right]},
$$

with an approximation error: $\Delta \chi=\Delta T /\left(T_{s}-\left.T_{\text {surf }}\right|_{\substack{k_{s}=0 \\ \theta=\pi / 2}}\right)<0.03$.

Derivation of Eqs. (2.13) and (2.14) is presented in Appendix A. Figure 6 represents approximating curves (2.13) and (2.14) together with computer simulation points taken from Figure 5.

\subsection{Two stages of evaporation of a sessile droplet}

In the presence of contact angle hysteresis the whole duration of the evaporation process can be subdivided into four stages. Below we consider only stages (I) and (II), which we refer below as stage one and two.

(I) The first stage: the radius of the contact line, $L$, remains constant and equal to its initial value $L_{0}$, which is the maximum value of the radius of the contact line. At the same time the contact angle, $\theta$, decreases from its initial value, which is equal to a static advancing contact angle, $\theta_{a d}$, to the final value, which is a static receding contact angle, $\theta_{r}$. The moment when the first stage started is adopted below as a zero moment, $t=0$. At this moment the radius of the contact line reaches its maximum value and the contact angle is equal to the static advancing contact angle, $\theta_{a d}$. 
(II) The second stage: the contact angle remains constant and equal to $\theta_{r}$, while the radius of the contact line decreases from $L_{0}$ to almost zero value.

We assume that during both stages of evaporation the droplet remains the spherical shape. That is, the volume of the droplet, $V$, can be presented as follows:

$$
V=L^{3} f(\theta), \quad f(\theta)=\frac{\pi}{3} \frac{(1-\cos \theta)^{2}(2+\cos \theta)}{\sin ^{3} \theta} .
$$

According to our previous consideration during both stages of evaporation the mass variation law has the form given by Eq. (2.2). Taking into account Eqs. (2.3) and (2.4) the parameter $\beta$ is adopted as a constant.

\subsubsection{The first stage of evaporation}

During this stage of evaporation the radius of the contact line remains constant and Eq. (2.2) can be rewritten as $L_{0}^{3} f^{\prime}(\theta) \frac{d \theta}{d t}=-\beta F(\theta) L_{0}$, or:

$$
L_{0}^{2} f^{\prime}(\theta) \frac{d \theta}{d t}=-\beta F(\theta),
$$

with the initial condition

$$
\left.\theta\right|_{t=0}=\theta_{a d}
$$

Let us introduce the following dimensionless time $\tau=t / t_{c h}$, where $t_{c h}=L_{0}^{2} / \beta$ is the characteristic time of the process. Eq. (2.16) now takes the following form:

$$
f^{\prime}(\theta) \frac{d \theta}{d \tau}=-F(\theta)
$$

Direct integration of the latter equation with the boundary condition (2.17) results in

$$
A\left(\theta, \theta_{a d}\right)=\tau,
$$

where $A\left(\theta, \theta_{a d}\right)=\int_{\theta}^{\theta_{a d}} \frac{f^{\prime}(\theta)}{F(\theta)} d \theta$. The latter equation shows that the deduced dependency should be universal and does not depend on the nature of the liquid and the droplet volume. The only parameter left is the initial contact angle (static advancing contact angle), which is supposed to be independently determined.

The first stage proceeds until the contact angle reaches its final value equal to the static receding contact angle. Using Eq. (2.18) we conclude that the end of the first stage, $\tau_{r}=t_{r} / t_{c h}$, is determined as

$$
A\left(\theta_{r}, \theta_{a d}\right)=\tau_{r}
$$

\subsubsection{The second stage of evaporation}

During that stage the contact angle remains constant but the radius of the contact line varies. Hence Eq. (2.2) can be rewritten now as $3 L^{2} f\left(\theta_{r}\right) \frac{d L}{d t}=-\beta F\left(\theta_{r}\right) L$. Let us introduce the same dimensionless time as before and dimensionless radius of the contact line: $\ell=L / L_{0}$. Hence the latter equation can be rewritten as

$$
\frac{d \ell^{2}}{d \tau}=-\frac{2}{3} \frac{F\left(\theta_{r}\right)}{f\left(\theta_{r}\right)}, \quad \tau>\tau_{r}
$$

with the following initial condition: $\ell\left(\tau_{r}\right)=1$. Direct integration of the latter equation results in $\ell^{2}(\tau)=1-\frac{2}{3} \frac{F\left(\theta_{r}\right)}{f\left(\theta_{r}\right)}\left(\tau-\tau_{r}\right)$, or:

$$
\ell(\tau)=\sqrt{1-\frac{2}{3} \frac{F\left(\theta_{r}\right)}{f\left(\theta_{r}\right)}\left(\tau-\tau_{r}\right)} .
$$


The latter dependence gives a universal dependence during the second stage of evaporation.

Resulting curves are schematically represented in Figure 7. Second stage of evaporation (when $\theta=\theta_{r}$ ) is usually finished before $\ell$ reaches zero, and the final point of this stage must belong to curves in Figure 7. Afterwards, the last stage of evaporation starts, when both $\ell$ and $\theta$ are decreasing over time, but this stage is not covered by present model.

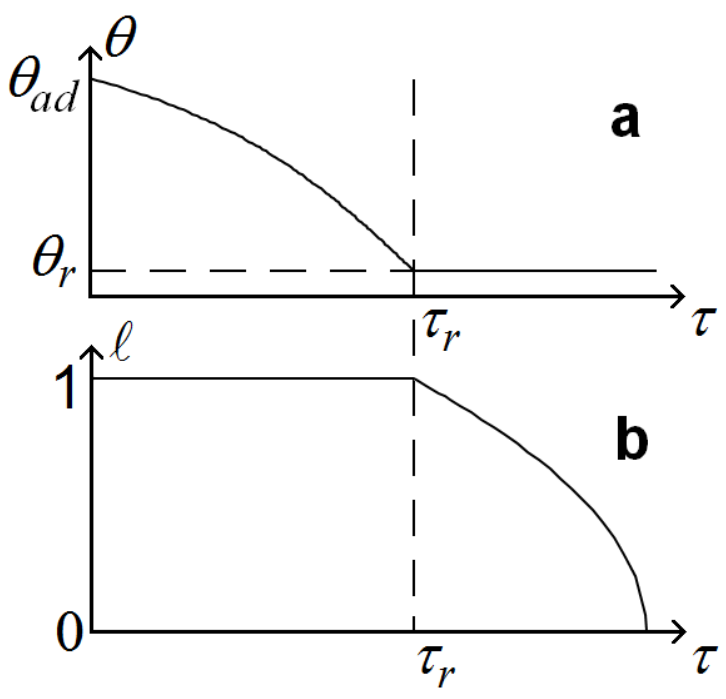

FiguRE 7. Dependencies on the dimensionless time: (a) contact angle, (b) radius of the contact line.

\section{Validation against experimental data}

Universal laws of evaporation of a sessile droplet represented by Eqs. (2.18) and (2.20) are validated against available experimental data extracted from literature sources [5, 7, 45]. The characteristic time of the process can be calculated using Eq. (2.19): $t_{c h}=t_{r} / A\left(\theta_{r}, \theta_{a d}\right)$. The latter can be calculated using experimental values of $\theta_{a d}$ and $\theta_{r}$. Obtained value of $t_{c h}$, as well as $\theta_{a d}$ and $\theta_{r}$ can be used to plot the dependencies $\theta(\tau)$ and $\ell(\tau)$ corresponding to the first (Figure 8) and second (Figure 9) stages of evaporation respectively. Figures 8 and 9 show good agreement of proposed theory and available experimental data.

Using the proposed above theory we can estimate the average temperature, $T_{a v}$, of the droplet's surface. Substituting the experimentally calculated value of $t_{c h}=t_{r} / A\left(\theta_{r}, \theta_{a d}\right)$ into the theoretical equation $t_{c h}=L_{0}^{2} / \beta$, and using the initial value of contact line radius, $L_{0}$, we can obtain the value of $\beta$. After that using Eq. (2.3) for $\beta$ and the dependence of concentration of saturated vapour, $c_{\text {sat }}(T)$, on temperature (Clausius-Clapeyron equation) [42] we can calculate the average temperature, $T_{a v}$, of the droplet's surface. Thus for the experimental data represented in Figures 8 and 9 we calculated the following values of $T_{a v}$ : for water on PMMA $T_{a v}=T_{\infty}-2.76 \mathrm{~K}$, for water on PET $T_{a v}=T_{\infty}-0.52 \mathrm{~K}$. Average temperatures of the droplet surface, $T_{a v}$, calculated using the above algorithm, are smaller than the ambient temperature, $T_{\infty}$, what indicates the surface cooling. We can see that calculated temperature drops, $T_{a v}-T_{\infty}$, are of the same order of magnitude as the experimentally measured temperature drop inside the droplet bulk [10] (see Figure 4). This confirms the validity of the proposed assumptions.

For the experiments presented in paper [5] (water on polished epoxy surface) it is impossible to calculate $T_{a v}$ because the humidity of ambient air was not specified in their experiments. For water on corning 


\section{$\theta, \mathrm{rad}$}

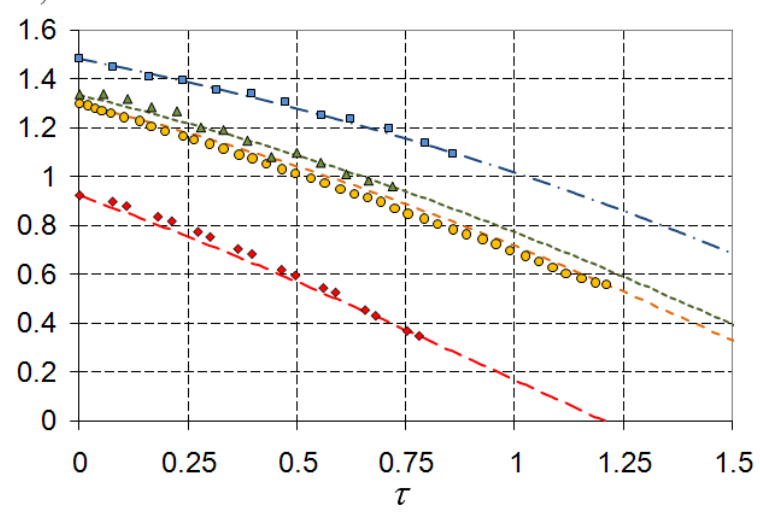

- Water on polished epoxy surface [5]

- Water on corning glass $7740[7]$

$\Delta$ Water on PMMA [45]

ם Water on PET [45]

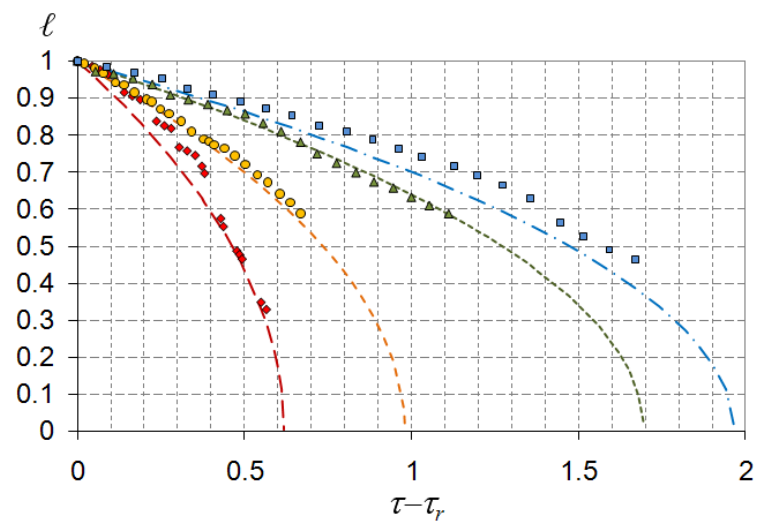

- Water on polished epoxy surface [5] - Theory $\left(\theta_{r}=0.348 ; t_{c h}=1267 \mathrm{~s}\right)$

- Water on corning glass $7740[7] \quad--$ Theory $\left(\theta_{r}=0.560 ; t_{c h}=911 \mathrm{~s}\right)$

$\triangle$ Water on PMMA [45]

- - - Theory $\left(\theta_{a d}=1.301 ; t_{c h}=911 \mathrm{~s}\right) \square$ Water on PET [45]

---- Theory $\left(\theta_{a d}=1.336 ; t_{c h}=1083 \mathrm{~s}\right)$

- - Theory $\left(\theta_{a d}=1.486 ; t_{c h}=760 \mathrm{~s}\right)$

Figure 9. Second stage of evaporation, Eq. (2.20). Dependence of dimensionless radius of contact line, $\ell$, on dimensionless time, $\tau$. $\tau_{r}$ is the duration of the first stage.
--- Theory $\left(\theta_{r}=0.959 ; t_{c h}=1083 \mathrm{~s}\right)$
$-\cdot-$ Theory $\left(\theta_{r}=1.096 ; t_{c h}=760 \mathrm{~s}\right)$
Figure 8. First stage of evaporation, Eq. (2.18). Dependence of contact angle, $\theta$, on dimensionless time, $\tau$.

glass, the estimation showed that the average temperature of the droplet surface, $T_{a v}$, appeared to be higher than the ambient temperature, $T_{\infty}$, what is in contradiction with the physics of the phenomenon. But our calculations show that taking relative air humidity $40 \%$ or less (instead of $60 \%$ reported by authors [7]) gives $T_{a v}<T_{\infty}$, as it should be. The latter result leads us to the conclusion that the relative humidity in experiments by Chen et al. [7] is measured inaccurately, and its value can be much different from the actual one in their experiments.

Note, real substrate used in any experiment is of a finite size, and calculated temperature drop, $T_{a v}-T_{\infty}$, can be used for the estimation of thermal conductivity of an equivalent semi-infinite substrate (see Figure 3), which gives the same cooling as real substrate of finite thickness.

Let us introduce new dimensionless times. Eq. (2.18) can be rewritten as:

$$
\int_{\theta}^{\pi / 2} \frac{f^{\prime}(\theta)}{F(\theta)} d \theta=\tau+\int_{\theta_{a d}}^{\pi / 2} \frac{f^{\prime}(\theta)}{F(\theta)} d \theta
$$

or

$$
B(\theta)=\tilde{\tau},
$$

where $B(\theta)=\int_{\theta}^{\pi / 2} \frac{f^{\prime}(\theta)}{F(\theta)} d \theta=A(\theta, \pi / 2)$, and $\tilde{\tau}=\tau+B\left(\theta_{a d}\right)$ is a new dimensionless time. Function $A\left(\theta_{1}, \theta_{2}\right)$ now can be expressed as follows: $A\left(\theta_{1}, \theta_{2}\right)=B\left(\theta_{1}\right)-B\left(\theta_{2}\right)$. According to its derivation Eq. (3.1) is supposed to be the universal curve describing the first stage of evaporation. The latter is completely confirmed by comparison with available experimental data in Figure 10. For the range of 
contact angle $0 \leq \theta \leq 2.967$ the dependence $B(\theta)$ can be approximated with the following function:

$$
B(\theta) \approx\left\{\begin{array}{ll}
\frac{-0.3714 \cdot \theta^{3}+0.3921 \cdot \theta^{2}-1.358 \cdot \theta+2.623}{-37.13 \cdot \theta^{5}+358.5 \cdot \theta^{4}-1387 \cdot \theta^{3}+2680 \cdot \theta^{2}-} & , 0 \leq \theta \leq \pi \\
-2585 \cdot \theta+995.9 & , \pi<\theta \leq 2.618 \\
\hline-8.404 \cdot 10^{4} \cdot \theta^{5}+1.151 \cdot 10^{6} \cdot \theta^{4}-6.302 \cdot 10^{6} \cdot \theta^{3} & \\
+1.726 \cdot 10^{7} \cdot \theta^{2}-2.363 \cdot 10^{7} \cdot \theta+1.294 \cdot 10^{7} & , 2.618<\theta \leq 2.967
\end{array} .\right.
$$

Hence,

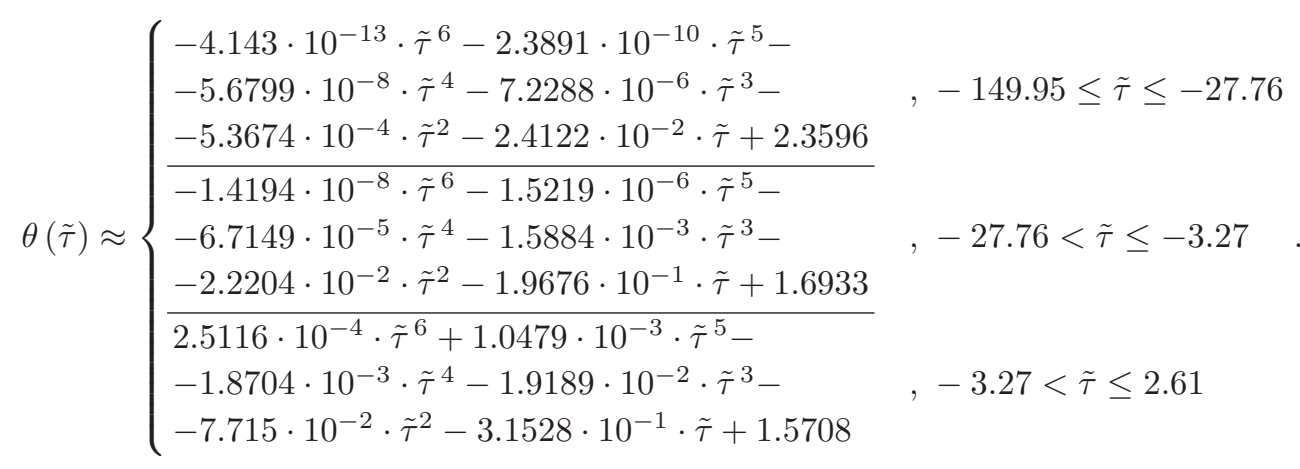

\section{$\theta$, rad}

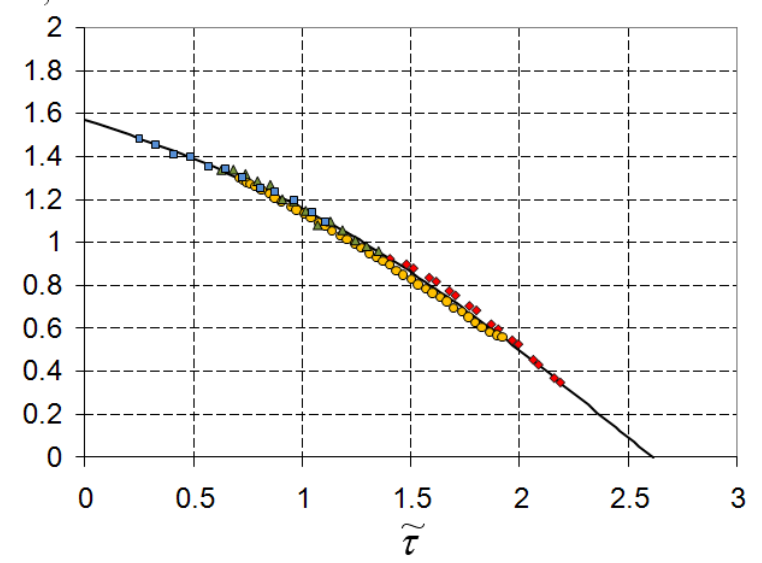

- Water on polished epoxy surface $[5]$ $\Delta$ Water on PMMA [45]
Figure 10. First stage of evaporation, Eq. (3.1). Dependence of contact angle, $\theta$, on dimensionless time, $\tilde{\tau}$.

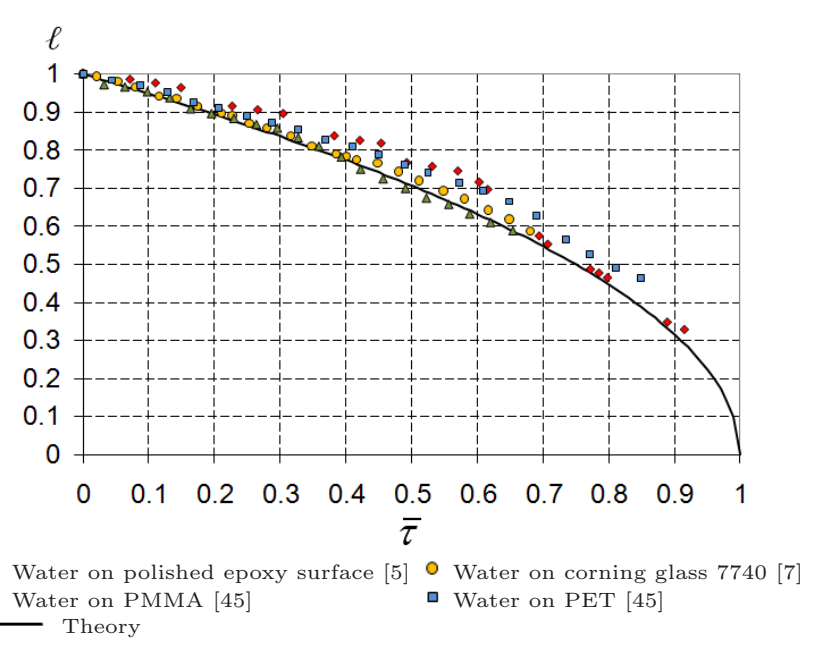

Figure 11. Second stage of evaporation, Eq. (3.2). Dependence of dimensionless radius of contact line, $\ell$, on dimensionless time, $\bar{\tau}$.

Introduction of the new dimensionless time $\bar{\tau}=\frac{2 F\left(\theta_{r}\right)}{3 f\left(\theta_{r}\right)}\left(\tau-\tau_{r}\right)$ to the Eq. (2.20) gives:

$$
\ell(\bar{\tau})=\sqrt{1-\bar{\tau}} .
$$

Again according to its derivation Eq. (3.2) is supposed to represent the universal curve describing the second stage of evaporation. Experimental data in Figure 11 confirm that conclusion. 


\section{Conclusion}

In this paper we presented a theory describing the evaporation of small sessile water droplets. In the presence of contact angle hysteresis the evaporation process can be subdivided into four stages. Introducing a dimensionless contact line radius and dimensionless times allowed us to deduce universal laws describing two stages of the evaporation process in the case of contact angle hysteresis. As the dependencies presented here are based on results of our computer simulations for water droplets, they can be considered as universal ones only for water based solutions. Though, it is quite possible that they are applicable for a wide range of liquids. The theory describes two stages ( $L=$ const at $1^{\text {st }}$ stage, and $\theta=\theta_{r}=$ const at $2^{\text {nd }}$ stage) and shows good agreement with experimental data for water available in the literature. In order to check if these dependencies are valid for liquids other than water, it is necessary to carry out additional studies.

Acknowledgements. This research was supported by the European Union under Grant MULTIFLOW, FP7-ITN2008-214919. V.M. Starov's research was also supported by the Engineering and Physical Sciences Research Council, UK (Grant EP/D077869/1). The work of R.G. Rubio was supported in part by the Spanish Ministerio de Ciencia e Innovacion through grant FIS2009-14008-C02-01, and by ESA through project MAP-AO-00-052. Both V.M. Starov and R.G. Rubio recognise a support from European Space Agency (PASTA project).

\section{Appendix A}

Let us find a semi-empirical approximation for the results of computer simulation presented in Figure 5. If we consider a specific case $k_{s}=0$, and accept the assumption that temperature of the droplet's surface, $T_{\text {surf }}$, is uniform and equal to its average temperature $T_{a v}$, then the solution of Laplace equation for temperature in air, $\Delta T=0$, gives the following total heat flux, $J_{h}$, from ambient air to the droplet surface:

$$
J_{h}=2 \pi k_{a}\left(T_{\infty}-T_{a v}\right) F(\theta) L .
$$

By analogy with electrical circuits temperature can be considered as the analogue of electrical potential, and total heat flux as the analogue of electrical current. Then it follows from the above formula (A.1) that analogue of the electrical resistance of air domain is

$$
R_{a}=\frac{T_{\infty}-T_{a v}}{J_{h}}=\frac{1}{2 \pi k_{a} F(\theta) L} .
$$

The analogue of electrical resistance of the substrate can be represented as

$$
R_{s}=\frac{1}{2 \pi k_{s} F(0) L}=\frac{1}{4 k_{s} L}
$$

To calculate the analogue of the resistance for water droplet, we assume for simplicity that total heat flux through the droplet:

$$
J_{h, w} \approx \bar{j}_{h, w} \cdot \pi L^{2} \approx k_{w} \frac{\Delta T_{w}}{\bar{h}} \cdot \pi L^{2},
$$

where $\bar{j}_{h, w}$ is the average density of the vertical component of the heat flux through the water droplet, $\Delta T_{w}$ is the temperature difference between bottom and top surfaces of the droplet, and $\bar{h}$ is the average height of the droplet, which can be represented as the ratio of droplet volume, $V$, and the area of droplet base: $\bar{h}=V /\left(\pi L^{2}\right)$. According to Eq. (2.15), droplet volume can be represented as $V=L^{3} f(\theta)$. Thus from Eq. (A.2) we get:

$$
R_{w} \approx \frac{\Delta T_{w}}{J_{h, w}} \approx \frac{V}{\pi^{2} L^{4} k_{w}}=\frac{f(\theta)}{\pi^{2} L k_{w}}
$$


By analogy with electrical circuits, the total heat flux to the droplet's surface with temperature $T_{a v}$ comprises the flux from ambient air with temperature $T_{\infty}$ and the flux (through the water droplet) from the substrate with far-field temperature $T_{s}$ :

$$
J_{h}=\frac{T_{\infty}-T_{a v}}{R_{a}}+\frac{T_{s}-T_{a v}}{R_{s}+R_{w}} .
$$

Using boundary condition $J_{h}=J \Lambda=J_{\pi / 2} F(\theta) \Lambda$, from Eq. (A.3) we get:

$$
T_{a v}=\frac{T_{\infty} / R_{a}+T_{s} /\left(R_{w}+R_{s}\right)-J_{\pi / 2} F(\theta) \Lambda}{1 / R_{a}+1 /\left(R_{w}+R_{s}\right)} .
$$

From Eq. (A.4) follows:

$$
\left.T_{\text {surf }}\right|_{\substack{k_{s}=0 \\ \theta=\pi / 2}}=\lim _{\substack{k_{s} \rightarrow 0 \\ \theta \rightarrow \pi / 2}} T_{a v}=T_{\infty}-\left.J_{\pi / 2} \Lambda R_{a}\right|_{\theta=\pi / 2} .
$$

Note that

$$
\left.R_{a}\right|_{\theta=\pi / 2}=\frac{1}{2 \pi k_{a} F(\pi / 2) L}=\frac{1}{2 \pi k_{a} L}=F(\theta) R_{a} .
$$

Combining equations (A.4), (A.5), and (A.6) we can calculate dimensionless temperature:

$$
\chi=\frac{T_{a v}-\left.T_{\text {surf }}\right|_{\substack{k_{s}=0 \\ \theta=\pi / 2}}}{T_{s}-\left.T_{\text {surf }}\right|_{\substack{k_{s}=0 \\ \theta=\pi / 2}}}=\frac{1}{1+R_{w} / R_{a}+R_{s} / R_{a}} .
$$

As the resistances are calculated roughly, we add empirical functions $\omega_{w}(\theta)$ and $\omega_{s}(\theta)$ into Eq. (A.7) to improve approximation:

$$
\chi=\frac{1}{1+\omega_{w}(\theta) R_{w} / R_{a}+\omega_{s}(\theta) R_{s} / R_{a}} .
$$

Substituting expressions for resistances we get:

$$
\chi=\frac{1}{1+(2 / \pi) \omega_{w}(\theta) f(\theta) F(\theta) k_{a} / k_{w}+(\pi / 2) \omega_{s}(\theta) F(\theta) k_{a} / k_{s}},
$$

or

$$
\chi=\frac{1}{1+F(\theta)\left(\omega_{1}(\theta) k_{a} / k_{w}+\omega_{2}(\theta) k_{a} / k_{s}\right)},
$$

where $\omega_{1}(\theta)=(2 / \pi) \omega_{w}(\theta) f(\theta)$ and $\omega_{2}(\theta)=(\pi / 2) \omega_{s}(\theta)$ are new empirical functions of contact angle. They have been chosen empirically:

$$
\begin{gathered}
\omega_{1}(\theta)=\sin \theta \\
\omega_{2}(\theta)= \begin{cases}\sin \theta-0.75 \cdot \theta+4.61, & \text { for } \quad T_{s}-T_{\infty}=0 \mathrm{~K} \\
\sin \theta-0.75 \cdot \theta+4.09, & \text { for } \quad T_{s}-T_{\infty}=5 \mathrm{~K}\end{cases}
\end{gathered}
$$

\section{Nomenclature}

Latin 


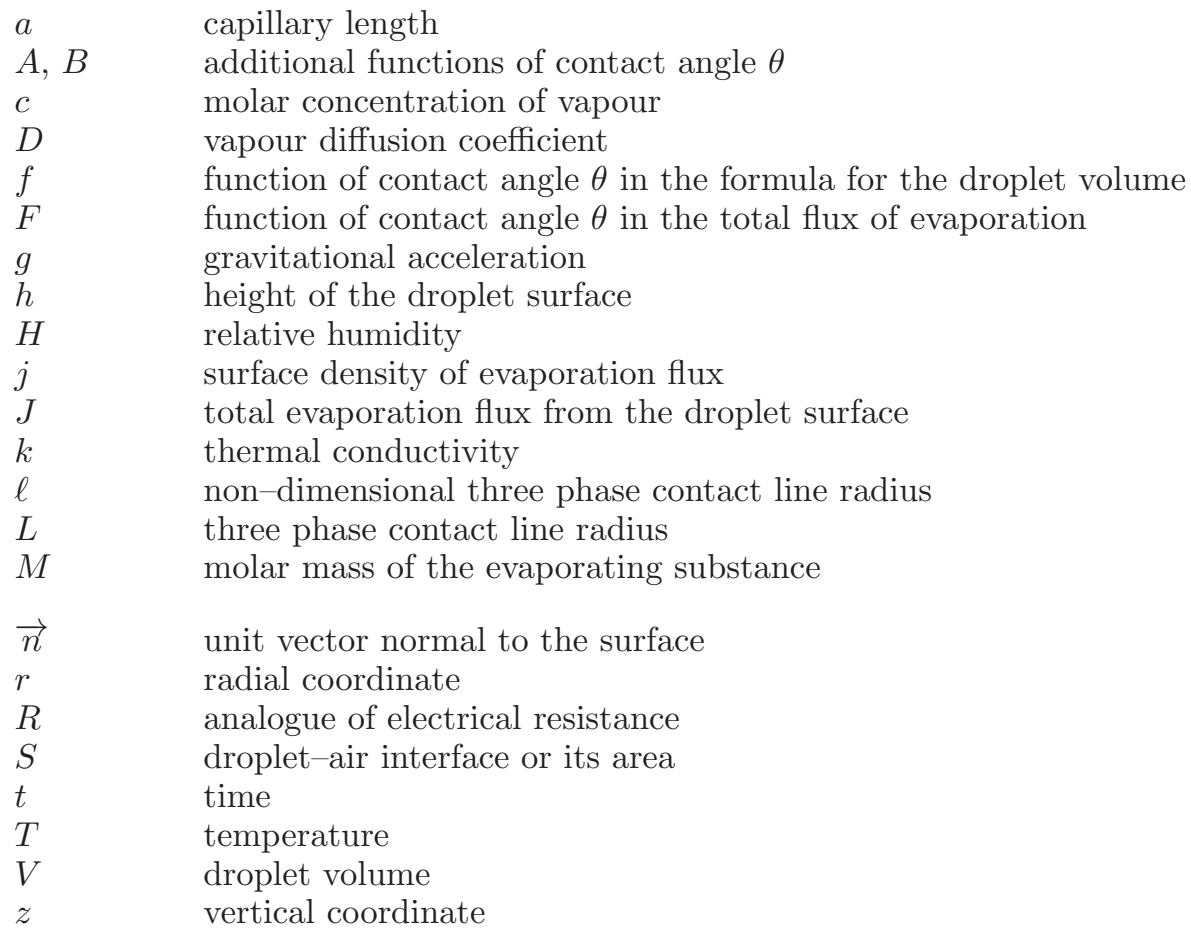

\section{Greek}

$\alpha$

$\beta$

$\gamma$

$\Delta$

$\theta$

$\Lambda$

$\rho$

$\tau, \bar{\tau}, \tilde{\tau}$

$\chi$

$\omega$

\section{Subscripts}

$\begin{array}{ll}\infty & \text { ambient value } \\ \pi / 2 & \text { corresponding to the contact angle } \theta=\pi / 2 \\ 0 & \text { initial value } \\ a & \text { air } \\ a d & \text { advancing (contact angle) } \\ a v & \text { average over the droplet's surface } \\ c & \text { molar } \\ c h & \text { characteristic } \\ h & \text { heat } \\ r & \text { receding (contact angle) } \\ s & \text { substrate } \\ \text { sat } & \text { saturated } \\ \text { surf } & \text { at the droplet surface } \\ w & \text { water }\end{array}$
proportionality coefficient in the expression for $J$ surface tension difference or error or Laplace operator contact angle latent heat of vaporization mass density non-dimensional time non-dimensional temperature empirical functions of contact angle

angle between $\vec{n}$ at the droplet surface and horizontal axis 


\section{References}

[1] D.C. Agrawal, V.J. Menon. Surface tension and evaporation: an empirical relation for water. Physical Review A, 46 (1992), 2166-2169.

[2] V.S. Ajaev. Spreading of thin volatile liquid droplets on uniformly heated surfaces. J. Fluid Mech. 528 (2005), $279-296$.

[3] D. Bensimon, A. Bensimon, F. Heslot. Process for aligning macromolecules by passage of a meniscus and applications. Patent No.: US 7754425 B2, (2010).

[4] R. Bhardwaj, X. Fang, D. Attinger. Pattern formation during the evaporation of a colloidal nanoliter drop: a numerical and experimental study. New J. Phys. 11 (2009), 075020.

[5] C. Bourges-Monnier, M.E.R. Shanahan. Influence of evaporation on contact angle. Langmuir 11 (1995), $2820-2829$.

[6] P.G. Campbell, L.E. Weiss. Tissue engineering with the aid of inkjet printers. Expert Opin. Biol. Ther. 7 (2007), 1123-1127.

[7] C.-T. Chen, F.-G. Tseng, C.-C. Chieng. Evaporation evolution of volatile liquid droplets in nanoliter wells. Sens. and Actuators A 130-131 (2006), 12-19.

[8] W.-L. Cheng, F.-Y. Han, Q.-N. Liu, R. Zhao, H.-L. Fan. Experimental and theoretical investigation of surface temperature non-uniformity of spray cooling. Energy (2010), doi:10.1016/j.energy.2010.10.044.

[9] R.V. Craster, O.K. Matar, K. Sefiane. Pinning, retraction, and terracing of evaporating droplets containing nanoparticles. Langmuir 25 (2009), 3601-3609.

[10] S. David, K. Sefiane, L. Tadrist. Experimental investigation of the effect of thermal properties of the substrate in the wetting and evaporation of sessile drops. Colloids Surf. A: Physicochem. Eng. Aspects 298 (2007), 108-114.

[11] R.D. Deegan. Pattern formation in drying drops. Phys. Rev. E 61 (2000), 475-485.

[12] R.D. Deegan, O. Bakajin, T.F. Dupont, G. Huber, S.R. Nagel, T.A. Witten. Contact line deposits in an evaporating drop. Phys. Rev. E 62 (2000), 756-765.

[13] V. Dugas, J. Broutin, E. Souteyrand. Droplet evaporation study applied to DNA chip manufacturing. Langmuir 21 (2005), 9130-9136.

[14] G.J. Dunn, S.K. Wilson, B.R. Duffy, S. David, K, Sefiane. A mathematical model for the evaporation of a thin sessile liquid droplet: comparison between experiment and theory. Colloids Surf. A: Physicochem. Eng. Aspects 323 (2008), $50-55$.

[15] G.J. Dunn, S.K. Wilson, B.R. Duffy, S. David, K. Sefiane. The strong influence of substrate conductivity on droplet evaporation. J. Fluid Mech. 623 (2009), 329-351.

[16] S.B. Fuller, E.J. Wilhelm, J.M. Jacobson. Ink-jet printed nanoparticle microelectromechanical systems. J. MEMS 11 (2002), 54-60.

[17] K.P. Galvin. A conceptually simple derivation of the Kelvin equation. Chem. Eng. Sci. 60 (2005), 4659-4660.

[18] B.-J. de Gans, P.C. Duineveld, U.S. Schubert. Inkjet printing of polymers: state of the art and future developments. Adv. Mater. 16 (2004), 203-213.

[19] F. Girard, M. Antoni, K. Sefiane. On the effect of Marangoni flow on evaporation rates of heated water drops. Langmuir 24 (2008), 9207-9210.

[20] F. Girard, M. Antoni, S. Faure, A. Steinchen. Evaporation and Marangoni driven convection in small heated water droplets. Langmuir 22 (2006), 11085-11091.

[21] F. Girard, M. Antoni, S. Faure, A. Steinchen. Numerical study of the evaporating dynamics of a sessile water droplet. Microgr. Sci. Technol. XVIII-3/4 (2006), 42-46.

[22] F. Girard, M. Antoni, S. Faure, A. Steinchen. Influence of heating temperature and relative humidity in the evaporation of pinned droplets. Colloids Surf. A 323 (2008), 36-49.

[23] F. Girard, M. Antoni. Influence of substrate heating on the evaporation dynamics of pinned water droplets. Langmuir 24 (2008), 11342-11345.

[24] G. Guena, C. Poulard, M. Voue, J.D. Coninck, A.M. Cazabat. Evaporation of sessile liquid droplets. Colloids Surf. A 291 (2006), 191-196.

[25] H. Hu, R.G. Larson. Evaporation of a sessile droplet on a substrate. J. Phys. Chem. B 106 (2002), $1334-1344$.

[26] H. Hu, R.G. Larson. Analysis of the microfluid flow in an evaporating sessile droplet. Langmuir, 21 (2005), $3963-3971$.

[27] H. Hu, R.G. Larson. Analysis of the effects of Marangoni stresses on the microflow in an evaporating sessile droplet. Langmuir, 21 (2005), 3972-3980.

[28] Y.M. Hung, Q. Seng. Effects of geometric design on thermal performance of star-groove micro-heat pipes. Int. J. Heat Mass Transfer (2010), doi:10.1016/j.ijheatmasstransfer.2010.09.070.

[29] C. Ingrosso, J.Y. Kim, E. Binetti, V. Fakhfouri, M. Striccoli, A. Agostiano, M.L. Curri, J. Brugger. Drop-on-demand inkjet printing of highly luminescent CdS and CdSe@ZnS nanocrystal based nanocomposites. Microelectr. Eng. 86 (2009), 1124-1126.

[30] S. Karlsson, A. Rasmuson, I.N. Björn, S. Schantz. Characterization and mathematical modelling of single fluidised particle coating. Powder Technol. 207 (2011), 245-256.

[31] H. Kim, J. Kim. Evaporation characteristics of a hydrophilic surface with micro-scale and/or nano-scale structures fabricated by sandblasting and aluminum anodization. J. Micromech. Microeng. 20 (2010), 045008

[32] J.H. Kim, Wei-Xian Shi, R.G. Larson. Methods of stretching DNA molecules using flow fields. Langmuir 23 (2007), $755-764$.

[33] S.H. Ko, J. Chung, N. Hotz, K.H. Nam, C.P. Grigoropoulos. Metal nanoparticle direct inkjet printing for low-temperature 3D micro metal structure fabrication. J. Micromech. Microeng. 20 (2010), 125010. 
[34] Du Peng, Li Luhai, Zhao Wen, Leng Xian, Hu Xuwei. Study on the printing performance of coated paper inkjet ink. Adv. Mater. Res. 174 (2011), 358-361.

[35] R.G. Picknett, R. Bexon. The evaporation of sessile or pendant drops in still air. J. Colloid Interface Sci. 61 (1977), 336-350.

[36] A.Ye. Rednikov, P. Colinet. Truncated versus extended microfilms at a vapor-liquid contact line on a heated substrate. Langmuir 27(5) (2011), 1758-1769.

[37] W.D. Ristenpart, P.G. Kim, C. Domingues, J. Wan, H.A. Stone. Influence of substrate conductivity on circulation reversal in evaporating drops. Phys. Rev. Lett. 99 (2007), 234502.

[38] N. Savva, S. Kalliadasis. Dynamics of moving contact lines: a comparison between slip and precursor film models. Europhys. Lett. 94 (2011), 64004.

[39] N.C. Schirmer, S. Ströhle, M.K. Tiwari, D. Poulikakos. On the principles of printing sub-micrometer 3D structures from dielectric-liquid-based colloids. Adv. Funct. Mater. XX (2010), 1-8, DOI:10.1002/adfm.201001426.

[40] F. Schonfeld, K.H. Graf, S. Hardt, H.J. Butt. Evaporation dynamics of sessile liquid drops in still air with constant contact radius. Int. J. Heat Mass Transfer 51 (2008), 3696-3699.

[41] K. Sefiane, L. Tadrist. Experimental investigation of the de-pinning phenomenon on rough surfaces of volatile drops. Int. Commun. Heat Mass Transfer 33 (2006), 482-490.

[42] S. Semenov, V.M. Starov, R.G. Rubio, M.G. Velarde. Instantaneous distribution of fluxes in the course of evaporation of sessile liquid droplets: computer simulations. Colloids Surf. A: Physicochem. Eng. Aspects 372 (2010), 127-134.

[43] Wetting and spreading dynamics /Victor M. Starov, Manuel G. Velarde, Clayton J. Radke; Boca Raton, Fla., CRC/Taylor \& Francis, London, 2007.

[44] E. Sultan, A. Boudaoud, M.B. Amar. Evaporation of a thin film: diffusion of the vapour and Marangoni instabilities. J. Fluid Mech. 543 (2005), 183-202.

[45] H. Yildirim Erbil, G. McHale, S.M. Rowan, M.I. Newton. Determination of the receding contact angle of sessile drops on polymer surfaces by evaporation. Langmuir 15 (1999), 7378-7385. 